\title{
Percepción y valoración del proceso de revegetación por los actores del territorio: un estudio preliminar en el Sistema Ibérico
}

\author{
Teodoro Lasanta \\ Instituto Pirenaico de Ecología (CSIC) \\ fm@ipe.csic.es \\ Nuria Esther Pascual Bellido \\ Universidad de La Rioja. Departamento de Ciencias Humanas y Sociales \\ nuria-esther.pascual@unirioja.es
}

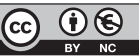

Recepción: febrero de 2014

Aceptación: junio de 2014

\section{Resumen}

Se estudia la percepción que tienen los actores (stakeholders) de Cameros Viejo (Sistema Ibérico) del proceso de revegetación, sus causas, consecuencias socioeconómicas e impactos en el medio natural. Se realizaron 25 entrevistas semiestructuradas. Los resultados muestran que perciben claramente que se ha producido un proceso de revegetación durante las últimas décadas, si bien difieren en las características de dicho proceso. Las respuestas son heterogéneas respecto a las causas, consecuencias e impactos que implica la revegetación. La población residente tiene una percepción más realista que la población foránea de los cambios en el medio natural. Se discute sobre el grado de correspondencia entre los cambios en el medio natural y la percepción que la sociedad tiene de ellos.

Palabras clave: campos abandonados; revegetación; conocimiento local; montaña mediterránea.

Resum. Percepció i valoració del procés de revegetació per part dels actors del territori: un estudi preliminar en el Sistema Ibèric

S'estudia la percepció que tenen els actors (stakeholders) de Cameros Viejo (Sistema Ibèric) del procés de revegetació, les seves causes, conseqüències socioeconòmiques i impactes en el medi natural. Es varen fer 25 entrevistes semiestructurades. Els resultats mostren que perceben clarament que s'ha donat un procés de revegetació durant les últimes dècades, tot i que difereixen en les característiques d'aquest procés. Les respostes són heterogènies respecte a les causes, consequiències i impactes que implica la revegetació. La població resident té una percepció més realista que la població forana dels canvis en el medi natural. Es discuteix sobre el grau de correspondència entre els canvis en el medi natural i la percepció que la societat en té.

Paraules clau: camps abandonats; revegetació; coneixement local; muntanya mediterrània. 
Résumé. Perception et évaluation du processus de revégétalisation pour les acteurs du territoire: une étude préliminaire dans le Système Ibérique

Cet article présente la perception des acteurs (stakeholders) de Cameros Viejo (Système Ibérique) concernant le processus de revégétalisation, ses causes, ses conséquences et les impacts socio-économiques sur l'environnement naturel. 25 entretiens semi-structurés ont été menés. Les résultats montrent clairement qu'il y a eu un processus de revégétalisation au cours des dernières décennies, même si les caractéristiques du processus different. Les réponses sont hétérogènes en ce qui concerne les causes, les conséquences et les répercussions de la revégétalisation. La population résidente présente une perception plus réaliste que la population extérieure concernant les changements de l'environnement naturel. Cet article examine le degré de correspondance entre les changements dans l'environnement naturel et la perception que la société en a.

Mots-clé: champs abandonnés; revégétalisation; connaissances locales; montagnes méditerranéennes.

Abstract. Perception and evaluation of the re-vegetation process by local stakeholders: a preliminary study in the Iberian Mountain Range

This paper analyses the perception of stakeholders in Cameros Viejo (Iberian Mountain Range, Spain) concerning the re-vegetation process, its causes, its consequences and the socioeconomic impacts on the natural environment. Twenty-five semi-structured interviews were conducted. The results clearly show that the population perceives there to have been a re-vegetation process in recent decades, although they differ in their ideas about the characteristics of the process. The answers are heterogeneous regarding the causes, consequences and impacts involved in re-vegetation. The resident population is more realistic than the foreign population about the perception of changes in the natural environment. Moreover, the degree of accordance between these environmental changes and public perceptions of the same is discussed.

Keywords: abandoned fields; re-vegetation process; local knowledge; Mediterranean mountains.

\author{
Sumario \\ 1. Introducción 4. Conclusiones e implicaciones \\ 2. Área de estudio y metodología Agradecimientos \\ 3. Resultados y discusión Referencias bibliográficas
}

\title{
1. Introducción
}

Las áreas rurales y el estilo de vida de los residentes en el medio rural han experimentado cambios en todo el mundo, con impactos diversos en los paisajes. Los procesos de industrialización, urbanización y las políticas de desarrollo rural han llevado, especialmente en los países desarrollados, a la polarización de la tierra utilizada: las áreas más productivas y con mejor accesibilidad a los centros económicos se han intensificado, mientras que las más alejadas o con tierras menos fértiles se han ido marginando y abandonando (MacDonald et al., 2000). 
El abandono de tierras agrícolas y de pastos ha favorecido procesos de revegetación espontánea en áreas de montaña, con la expansión de matorrales y bosques (Waisamen y Bliss, 2002; Keenleyside y Turker, 2010). En España, además, la revegetación tiene que ver con la política forestal implantada desde 1940, que llevó a la reforestación de grandes extensiones de tierras (Ortigosa, 1990; Vicente-Serrano et al., 2005; Lasanta y Vicente-Serrano, 2007). El avance de la vegetación borra algunos rasgos de los paisajes tradicionales, considerados con frecuencia como paisajes culturales (Lasanta et al., 2005; Gellrich et al., 2007). La revegetación tiene también otras consecuencias ecológicas, como la desaparición de paisajes de grano fino o en mosaico, lo que conduce a su simplificación y homogeneización, a la pérdida de muchos hábitats seminaturales y a la disminución de la biodiversidad (Henle et al., 2008; Stoate et al., 2009; Uematsu et al., 2010). Desde una perspectiva social, la desaparición de un paisaje en mosaico y, con ello, la pérdida de cualidades escénicas da lugar a la impresión de un territorio mal administrado, que genera en la sociedad sentimientos de desolación, aislamiento, opresión y de pérdida de contacto con el territorio (Benjamin et al., 2007; Bell et al., 2009). El abandono de campos y la revegetación tienen además destacadas influencias en la dinámica hidrológica y en la erosión del suelo (García-Ruiz y Lana-Renault, 2011).

Sin embargo, algunos autores ven el abandono de tierras como una oportunidad para la renaturalización (re-wilding) del paisaje. Ello favorecería la expansión de ungulados silvestres, que podrían contribuir al mantenimiento de un paisaje diverso y estable; papel que ha cumplido tradicionalmente la ganadería extensiva (Bowen et al., 2007). Navarro y Pereira (2012) señalan que el paisaje mediterráneo es más sostenible en un territorio más renaturalizado, al reducirse el riesgo de erosión de las laderas. Los mismos autores insisten en que la regeneración de los bosques podría prestar algunos servicios ecosistémicos, como incrementar el secuestro de carbono y su uso para fines recreativos. Por último, sugieren que los partidarios de mantener los usos del suelo tradicionales, sobre todo los agrícolas, subestiman el enorme trabajo humano que se necesita para su sostenimiento.

La demanda y los gustos de la sociedad por el paisaje están cambiando a medida que aumenta la permeabilidad entre la sociedad urbana y rural, y a la vez que los agricultores pierden peso económico y social (Domon, 2011). El concepto de paisaje multifuncional está ganando terreno entre los políticos, los científicos y la sociedad, buscando cierto equilibrio entre los aspectos económicos, ecológicos, sociales y las funciones culturales (Antrop, 2005; Sayadi et al., 2009). En este sentido, hay que recordar que las reformas de la política agraria comunitaria (PAC) han ido dando más peso, progresivamente, al desarrollo rural y a las prácticas agropecuarias más respetuosas con el medio ambiente. También porque el paisaje constituye un recurso cada vez más importante para el desarrollo de las zonas rurales (Hunziker et al., 2008). La multifuncionalidad del paisaje se ha convertido en una condición esencial para el desarrollo sostenible y para la gestión del territorio. Ahora se persigue la conservación 
de las cualidades y los valores inherentes del paisaje y el sostenimiento de las economías rurales (Antrop, 2006).

Dentro de este complejo proceso de transformación de la vida rural, no se pueden comprender las tendencias futuras de desarrollo y conservación sin conocer la opinión de los actores (stakeholders) de un territorio, que constituyen en realidad los artífices más influyentes en los cambios de paisaje (Kaur, et al., 2004; Bell et al., 2009). El objetivo de este trabajo es conocer la percepción que tienen los stakeholders de Cameros Viejo sobre el proceso de revegetación: sus características, sus causas y sus impactos socioeconómicos y ambientales. Se espera que su opinión pueda ayudar a interpretar el proceso de renaturalización y conocer mejor el impacto social del abandono de tierras. También puede contribuir a implementar las decisiones sobre desarrollo sostenible y adaptación al cambio global.

\section{2. Área de estudio y metodología}

\subsection{El área de estudio}

Este trabajo se llevó a cabo en Cameros Viejo, altos valles del Leza y Jubera (Figura 1), en el sector riojano del Sistema Ibérico (Latitud Norte de su punto central: $42^{\circ} 12^{\prime} 46^{\prime \prime}$ y Longitud Oeste $\left.2^{\circ} 25^{\prime} 5^{\prime \prime}\right)$. La superficie es de

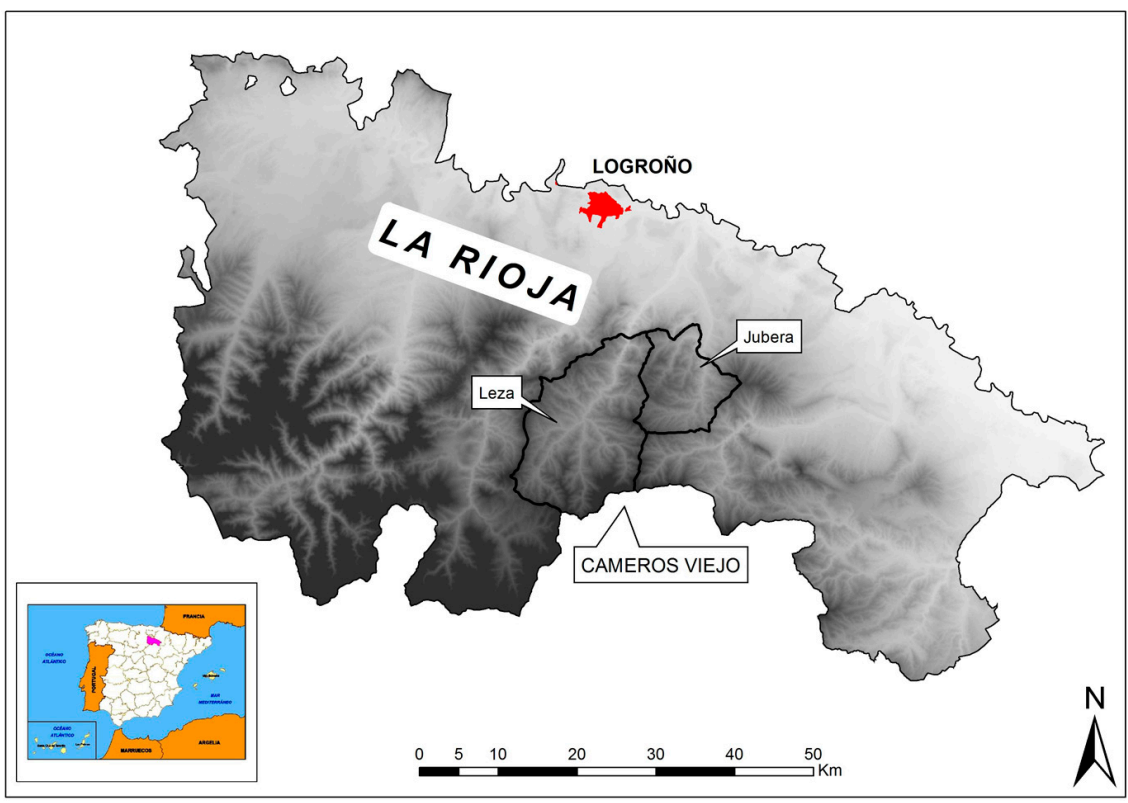

Figura 1. Área de estudio.

Fuente: elaboración propia. 
Tabla 1. Cambios en los usos y cubiertas del suelo en Cameros Viejo entre 1956 y 2001

\begin{tabular}{|c|c|c|c|c|c|}
\hline \multirow[b]{2}{*}{ Clases } & \multicolumn{2}{|c|}{ Año 1956} & \multicolumn{2}{|c|}{ Año 2001} & \multirow[b]{2}{*}{ \% cambio } \\
\hline & $\begin{array}{l}\text { Superficie } \\
\text { (ha) }\end{array}$ & $\begin{array}{c}\text { Superficie } \\
(\%)\end{array}$ & $\begin{array}{l}\text { Superficie } \\
\text { (ha) }\end{array}$ & $\begin{array}{c}\text { Superficie } \\
(\%)\end{array}$ & \\
\hline Espacio cultivado & $13.543,5$ & 34,49 & 0 & 0 & $-34,49$ \\
\hline Espacio agrícola abandonado & $2.424,7$ & 6,17 & 0 & 0 & $-6,17$ \\
\hline Frondosas & $4.434,2$ & 11,29 & $11.394,3$ & 29,01 & $+17,72$ \\
\hline Coníferas & 13,26 & 0,03 & 135,9 & 0,35 & $+0,32$ \\
\hline Bosque mixto & 0 & 0 & 50,5 & 0,13 & $+0,13$ \\
\hline Repoblación forestal & 76,1 & 0,19 & 4.278 & 10,89 & $+10,70$ \\
\hline Matorral & 17.154 & 43,68 & $22.951,5$ & 58,4 & $+14,72$ \\
\hline Pastizales & 882,4 & 2,25 & 19 & 0,05 & $-2,2$ \\
\hline Áreas erosionadas & 706,5 & 1,80 & 0 & 0 & $-1,80$ \\
\hline Otros usos & 36,2 & 0,092 & 441,6 & 1,12 & $+1,03$ \\
\hline
\end{tabular}

Fuente: elaboración propia a partir de las fotografías aéreas de 1956 y 2001.

$392,71 \mathrm{~km}^{2}$, distribuida por 15 municipios. En 2013 la población de derecho era de 1.223 habitantes, lo que equivale a una densidad de $3,1 \mathrm{hab} . / \mathrm{km}^{2}$. La población descendió a lo largo del siglo xx, ya que en 1900 había 8.013 habitantes; en 1950, 5.571, y en 1981, 1.477.

Desde el siglo XII se deforestaron tierras para favorecer las áreas de pastoreo con las que alimentar a los rebaños trashumantes en verano. En los siglos XVIII y XIX se roturaron muchas laderas para el cultivo de cereales con el fin de alimentar a la población local y sustituir a la economía ganadera y textil, ya en crisis tras el inicio de la decadencia de la trashumancia. Desde mediados del siglo xx la mayor parte de la población emigra, se abandona el espacio agrícola y se transforma el sistema de gestión ganadera, lo que genera un destacado proceso de sucesión vegetal (Lasanta y Errea, 2001). En la tabla 1 se comprueba que entre 1956 y 2001 desapareció el espacio agrícola, que hasta 1956 ocupaba $15.968,2$ ha, de las que $13.543,5$ se mantenían en cultivo y 2.424,7 se habían dejado de cultivar para 1956. Los bosques de frondosas aumentaron de $4.434,2$ ha a $11.394,3$, a partir de la expansión de pequeños bosquetes que se habían conservado en las cimas y áreas poco accesibles (Arnáez et al., 2009). Las coníferas se expandieron por la reforestación con pinos que realizó la administración; entre 1956 y 2001 se pasó de 76,1 a 4.278 ha. Los matorrales incrementaron su superficie, mientras que los pastizales y áreas erosionadas la vieron reducida.

En la cubierta vegetal actual dominan los bosques de Fagus silvatica y Quercus pirenaica en las cimas, mientras que a altitudes más bajas aparece Quercus rotundifolia sp. Valota (Figura 2). Algunas laderas están cubiertas por pinos de repoblación. Los matorrales de Genista scorpius (aliaga), Thymus vulgaris, Rosmarinus officinalis y Buxus sempervirens dominan en suelos calizos (Figura 3), mientras que en los silíceos Cistus laurifolius (jara) es la especie más representativa (Arnáez et al., 2009). La abundancia de matorrales (22.951,5 ha en 2001) 


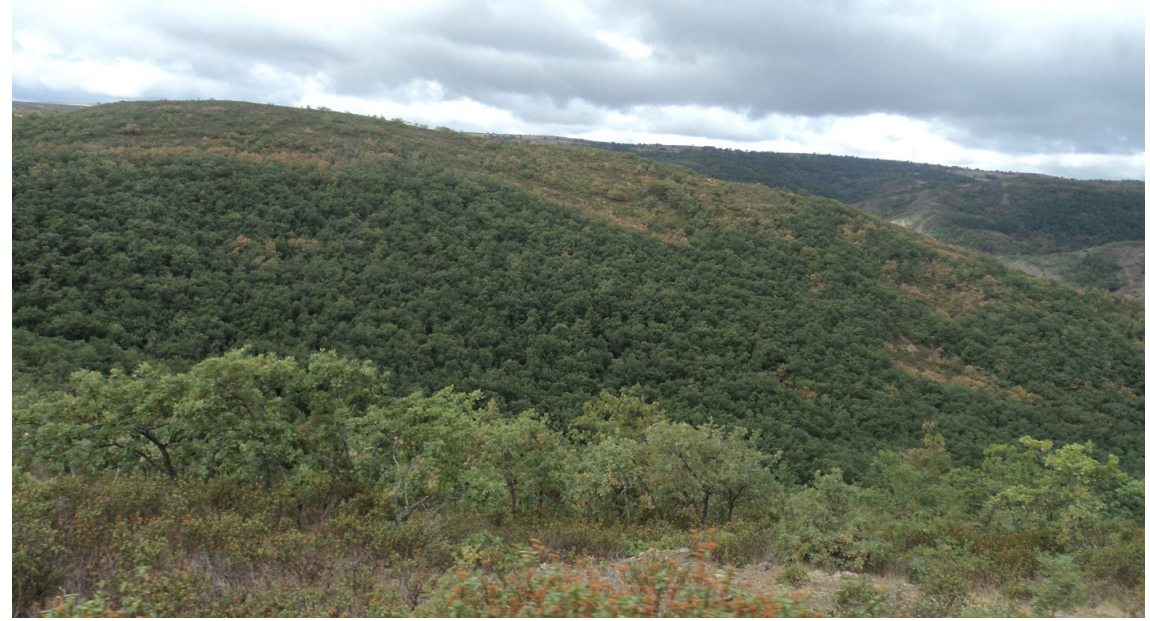

Figura 2. Revegetación de bosque natural en la aldea de San Martín (Valle del Jubera).

Fuente: elaboración propia.

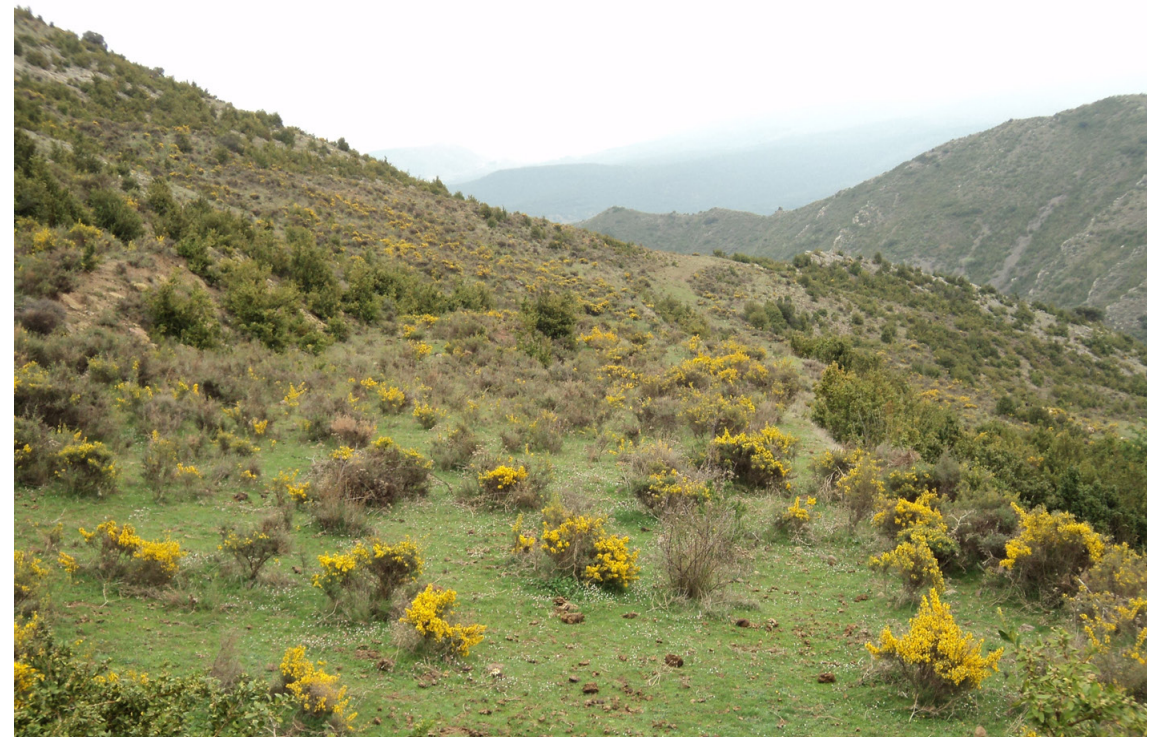

Figura 3. Colonización vegetal por Genista scorpius en la aldea de San Martín (Valle del Jubera).

Fuente: elaboración propia. 


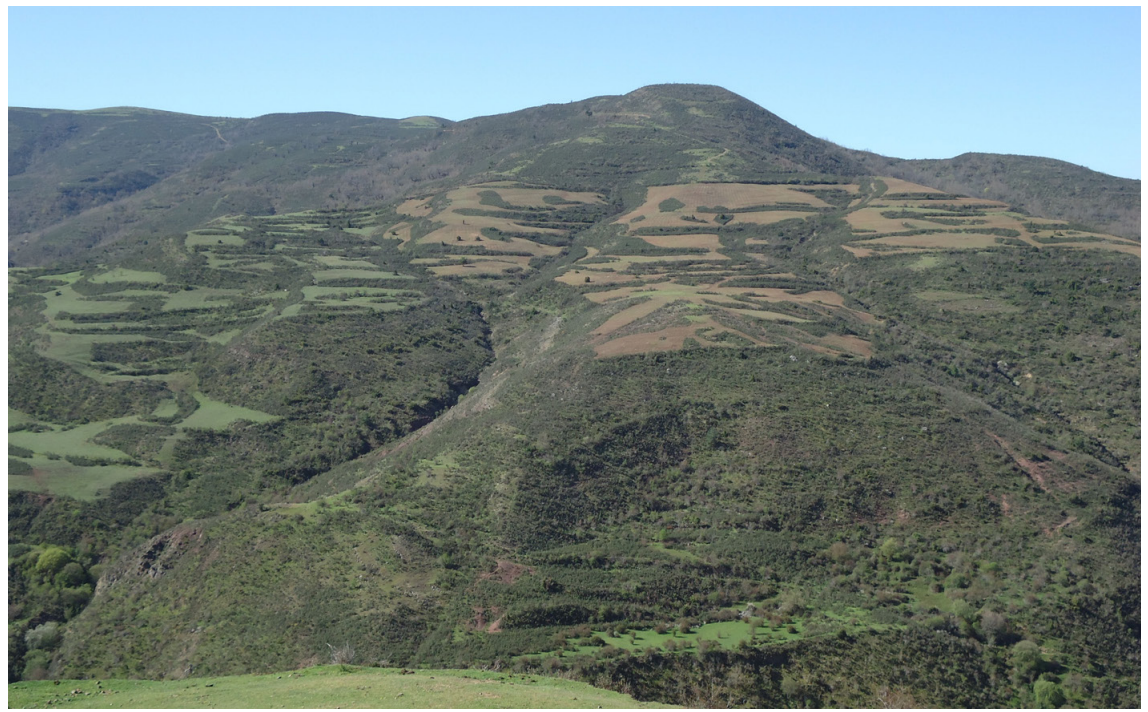

Figura 4. Imagen tras el desbroce de matorrales en una ladera de Soto en Cameros (Valle del Leza).

Fuente: elaboración propia.

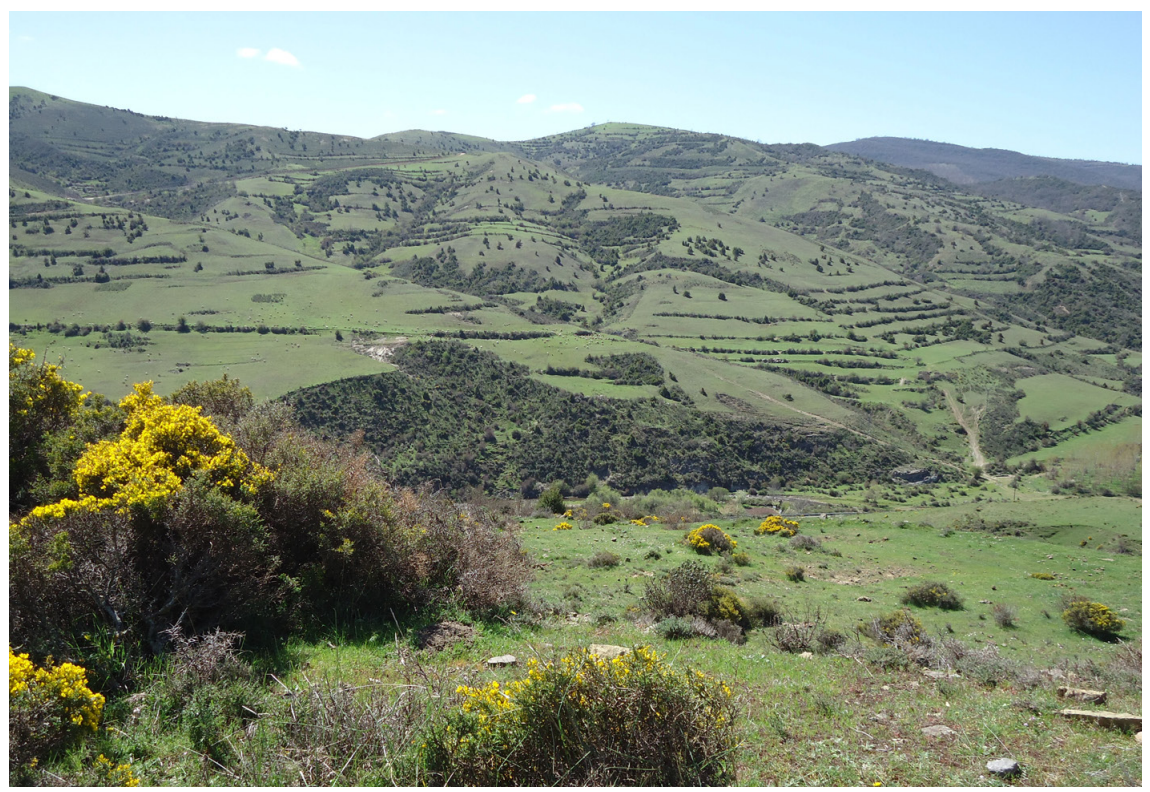

Figura 5. Ladera desbrozada de matorrales en Jalón de Cameros (Valle del Leza).

Fuente: elaboración propia. 
ha hecho que desde 1986 el Gobierno de La Rioja desbroce algunas laderas con el fin de regenerar pastos y controlar los incendios (Figuras 4 y 5). Entre 1986 y 2011 desbrozó el 22,2\% de la superficie de matorrales de la Sierra Riojana (Lasanta et al., 2013).

\subsection{Metodología}

Durante el mes de agosto de 2011 se realizaron 25 entrevistas semiestructuradas para conocer la valoración y percepción de los cambios de cubierta vegetal por los stakeholders, sus causas y sus impactos (Fernández-Giménez y Fillat, 2012). Se seleccionaron personas vinculadas con Cameros Viejo y de más de 40 años, con el fin de que pudieran tener una perspectiva temporal de los cambios. Con todos ellos se concertó una entrevista mediante una llamada de teléfono. Se dejó de realizar entrevistas cuando las adicionales ofrecían muy poca información nueva, y las respuestas resultaban reiterativas. Consideramos que con las 25 realizadas recogíamos la opinión de los actores más representativos y que realizar más entrevistas no compensaba el tiempo invertido. Las entrevistas duraron de 1 a 2 horas y se llevaron a cabo en el domicilio del entrevistado, en un lugar público de su pueblo o en el campo.

En la entrevista se preguntó por los datos personales del entrevistado: edad, profesión, nivel de estudios, su sensibilización medio ambiental, lugar de nacimiento y relación con el área de estudio, diferenciando entre personas que viven y trabajan en Cameros Viejo (grupo A), las que sólo trabajan en el área de estudio (grupo B), o las que poseen una segunda residencia (grupo C). El segundo bloque de preguntas se dirigió a conocer su opinión sobre los cambios de cubierta vegetal en las últimas décadas (extensión y tipo) y su alcance espacial y temporal. El tercer grupo de preguntas se orientó a conocer las causas del proceso de revegetación. En el cuarto bloque se les preguntó por las consecuencias socioeconómicas: en la agricultura, ganadería, paisaje, turismo, caza, recuperación de los pueblos... Por último, se preguntó sobre los posibles impactos ambientales de la revegetación en la erosión del suelo, en la cantidad y calidad del agua, en el riesgo de incendios y en la fauna.

\section{Resultados y discusión}

\subsection{Perfil de la población entrevistada}

En la tabla 2 se incluye el perfil de los entrevistados. Trece nacieron en Cameros Viejo y 12 fuera del área de estudio. La distribución por edades muestra más participación de los mayores: 8 superan los 70 años y de los «jóvenes», 8 no habían cumplido los 50 años. Entre ambos grupos se contó con la colaboración de 9 personas. En la estructura por sexo hay un claro desequilibrio, ya que sólo 4 son mujeres, cifra que refleja — aunque quizás en demasía— la mayor presencia de hombres en la zona. Dominan las personas con estudios primarios (12), si bien las otras dos categorías están bien representadas, especialmente la de las personas con estudios superiores. Este hecho se explica 
Tabla 2. Perfil de los agentes sociales entrevistados

\begin{tabular}{lcccc}
\hline Lugar de nacimiento & \multicolumn{2}{c}{ Cameros Viejo } & \multicolumn{2}{c}{ Fuera } \\
& \multicolumn{2}{c}{13} & \multicolumn{2}{c}{12} \\
\hline Edad & $40-50$ años & $50-60$ años & $60-70$ años & 70 años \\
& 8 & 4 & 5 & 8 \\
\hline Sexo & \multicolumn{2}{c}{ Varón } & \multicolumn{2}{c}{ Mujer } \\
& \multicolumn{2}{c}{21} & \multicolumn{2}{c}{ Superiores } \\
\hline Estudios & Primarios & Secundarios & 8 & \\
& 12 & 5 & Terciario & Sector público \\
Ocupación & Primario & Secundario & 4 & 12 \\
\hline Grupo social & 6 & 3 & C: 11 &
\end{tabular}

Fuente: elaboración propia a partir de entrevistas semiestructuradas.

porque se tuvo especial interés en conocer la opinión de los técnicos de la Administración que trabajan en el área de estudio (ingenieros, biólogos, veterinarios...). En la ocupación dominan los empleados del sector público, ya que también se entrevistó a guardas forestales, secretarios de ayuntamiento, empleados de la conservación del monte... Por último, en la tabla 2 se constata que 6 de los entrevistados se incluyen en el grupo A, 8 en el grupo B y 11 en el grupo C. El grupo A está constituido por personas mayores, con estudios primarios y ocupados en el sector primario. El grupo B por personas de mediana edad, con estudios superiores, que trabajan en el sector público. El grupo $\mathrm{C}$ es muy heterogéneo en todas las variables, ya que está compuesto por antiguos emigrantes y personas que han comprado una segunda residencia. La distribución del perfil de los entrevistados permite pensar que los principales agentes sociales de la zona están representados, por lo que los resultados obtenidos pueden considerarse —en nuestra opinión- válidos para conocer la percepción y valoración del proceso de revegetación.

\subsection{Caracteristicas del proceso de revegetación}

A la pregunta ¿existe proceso de revegetación?, la totalidad de los entrevistados respondieron que sí (Tabla 3), coincidiendo plenamente con lo que señala la información disponible: la superficie forestal se incrementó en 14.708 ha entre 1956 y 2001 (Arnáez et al., 2009). El 60\% de ellos considera que es un proceso intenso, mientras que sólo 1 persona lo ve escaso y 9 interlocutores (el $36 \%$ ), moderado. La mayoría de los entrevistados (17, el 68\%) opina que es generalizado por todo el territorio, mientras que 8 ven que se está produciendo en áreas concretas: antiguos campos de cultivo, preferentemente. Para 16 personas, matorrales y árboles conjuntamente están cubriendo mayores superficies progresivamente, mientras que 9 piensan que el bosque no avanza y que sólo se extienden los matorrales. Nadie opina que la revegetación es cosa exclusiva de árboles. Sobre en qué momento se inició la expansión de la cubierta vegetal, 
Tabla 3. Respuestas a la pregunta «¿Existe proceso de revegetación?»

\begin{tabular}{lllllll}
\hline ¿Existe proceso de revegetación? & Sí: 25 & \multicolumn{2}{l}{ No: 0} & & \\
\hline Grado & Intenso: 15 & Moderado: 9 & Escaso: 1 & \\
\hline Localización & Generalizado: 17 & \multicolumn{4}{l}{ Áreas concretas: 8} & \\
\hline Tipo & Matorral: 9 & Arbolado: 0 & Ambos: 16 & \\
\hline Inicio del proceso & $<10$ & $10-20$ & $20-30$ & $30-40$ & $40-50$ & $>50$ \\
& años: 0 & años: 1 & años: 2 & años: 8 & años: 6 & años: 8 \\
\hline
\end{tabular}

Fuente: elaboración propia a partir de entrevistas semiestructuradas.

una amplia mayoría considera que es un proceso ya dilatado en el tiempo, ya que 22 personas (88\%) piensan que hace más de 30 años, e incluso 8 opinan que hace más de 50 años. Fueron muchas las personas que señalaron que fue en los años sesenta, coincidiendo con la emigración masiva de la población y con una fuerte contracción del área agrícola, cuando los matorrales comenzaron a cubrir numerosas laderas.

Los resultados obtenidos en los estudios realizados en el área de estudio y en otras zonas de características parecidas muestran que el proceso de revegetación es complejo (Molinillo et al, 1997; Lasanta et al., 2000; Lasanta y Vicente-Serrano, 2003; Arnáez et al., 2011). Lo habitual es que se inicie nada más abandonarse un campo, pasando por distintas fases: cubierta de herbáceas, matorrales, bosque aclarado y bosque denso. La rapidez y extensión superficial de dicho proceso está condicionada por factores ambientales (clima, fertilidad del suelo, topografía) y humanos, destacando la gestión tras el abandono: existencia o no de pastoreo, incendios, desbroces... La complejidad del proceso explica que los entrevistados puedan tener distinta percepción. En este sentido, Soliva (2007) señala que en cada sistema social se constituyen grupos de individuos que tienen diferente percepción del paisaje, en función de sus gustos o los objetivos que pretenden alcanzar.

\subsection{Causas del proceso de revegetación}

A la hora de conocer las causas que la población entiende como responsables del proceso de revegetación hay un consenso bastante importante. Todos ellos (Tabla 4) consideran que la política de reforestación de la Administración es un factor determinante, así como la falta de ganado ( 24 personas) y la baja presión de la población sobre los productos del monte (23 personas). El resto de las causas parecen tener menos importancia. No obstante, 16 personas (el 64\%) consideran que el control de la Administración sobre la recogida de leñas y tala de árboles es un factor a tener en cuenta, ya que facilita la progresión de los matorrales y la densificación del sotobosque. Quince personas opinan que la prohibición de quemas prescritas o fuegos controlados para regenerar pastos, como se hacía anteriormente, es una causa para el avance de los matorrales; otros, sin embargo, consideran que ya no son necesarios los incendios, ya que la regeneración de pastos se obtiene mediante los desbroces realizados por la 
Tabla 4. Causas del proceso de revegetación

\begin{tabular}{lr}
\hline Falta de ganado & 24 \\
Falta de personas en el monte & 23 \\
No quemar & 14 \\
Cambio climático & 3 \\
Política de la Administración favorable a la caza & 1 \\
Control de la Administración en la recogida de leñas... & 16 \\
Propagación de semillas por la fauna & 11 \\
Repoblaciones forestales realizadas por la Administración & 25 \\
\hline
\end{tabular}

Fuente: elaboración propia a partir de entrevistas semiestructuradas.

Administración, y que tales desbroces cumplen el antiguo papel de los incendios, por lo que la ausencia de éstos no debe ser una causa que deba tenerse en cuenta. Resulta curioso reseñar que 11 personas consideran que la fauna (jabalíes, pájaros, ciervos...) pueden contribuir, mediante sus deyecciones, a dispersar semillas y, con ello, a favorecer la aparición de matorrales en áreas no cubiertas anteriormente. Sólo 3 personas (el 12\%) manifiestan que el cambio climático favorece la revegetación, mientras que el $88 \%$ restante considera que nada tiene que ver. Por último, 1 persona señaló que la Administración tiene interés en favorecer la caza mayor y, por ello, no controla la expansión de los matorrales.

Se ha señalado anteriormente que la principal causa de la revegetación para los stakeholders es la política de la Administración de repoblación forestal. La acusada degradación paisajística y, en muchos casos, los limitados recursos maderables implicaron una política de reforestación del territorio. Con las repoblaciones forestales se perseguía incrementar la producción maderera, para satisfacer la creciente demanda industrial, y restaurar la dinámica hidrológica, con el fin de reducir la erosión de los suelos, aprovisionar de agua a las cuencas y limitar la intensidad de las crecidas (Ortigosa et al., 1990). Lo cierto es que en Cameros Viejo se repoblaron 4.189 ha (el 9,3\% de su extensión), ocupando a veces términos completos de antiguos municipios (La Santa) o de pueblos (Santa María y Montalvo, por ejemplo). No es extraño, pues, que la población señale de forma unánime que la repoblación forestal es una causa.

La segunda causa más importante, según el número de respuestas, es la falta de ganado en el monte. Llevan razón, aunque hay que matizar la respuesta. Según Lasanta (2009), la carga ganadera de Cameros Viejo apenas cambió entre 1950 y 2005, por lo que no hay menos ganado ahora que hace cincuenta años. Sin embargo, sí que se ha producido una profunda modificación en la composición de la cabaña y en el sistema de gestión. La composición tradicional estaba constituida por ovino y caprino (76,7\% del censo en 1950$)$, mientras que en la reciente domina el vacuno ( $86,8 \%$ en 2005$)$ que, además, está compuesto por ejemplares de razas foráneas: pardo-alpina, charolesa, hereford, limousin... Por otro lado, el ganado tradicional era conducido por pastores, 
mientras que desde los años setenta pasta sin vigilancia de los ganaderos. Estos hechos han llevado a que tan sólo se aprovechen los pastos próximos a las naves ganaderas, puntos de pienso y abrevada y las áreas desbrozadas, mientras que la mayor parte de las laderas apenas son visitadas por el ganado. Ha habido, pues, un cambio en la presión sobre los pastos, con áreas utilizadas intensivamente y otras marginadas o abandonadas, en las que domina un proceso de sucesión vegetal, que en función de la etapa en la que se encuentre muestra laderas cubiertas por matorrales o por vegetación arbórea.

Otras causas importantes para los actores son la falta de personas en el monte, y la prohibición o, en su defecto, el control estricto sobre la recogida de leñas y matas para los hornos, así como sobre las quemas para regenerar pastos. Todas estas actividades formaban parte de la vida tradicional, contribuyendo a mantener limpio el monte y un paisaje con menos vegetación (Gaviria y Baigorri, 1984). Son menos los entrevistados que señalan otras causas: el cambio climático (sólo 3), una normativa favorecedora de la caza (1) y la distribución de semillas por la fauna (11).

\subsection{Consecuencias en el aprovechamiento del territorio}

Los entrevistados responden de forma muy diferente sobre las consecuencias del proceso de revegetación en los posibles aprovechamientos del territorio (Tabla 5). Una amplia mayoría (el 92\%) opina que no tiene ningún efecto sobre la actividad agrícola, dado que ésta se ha reducido a la mínima expresión. Sin embargo, 2 personas consideran que el proceso es positivo aduciendo que la bajada de los precios de la tierra agrícola puede favorecer la compra de campos e incrementar —especifican — la plantación de nogales y almendros. Lo curioso de la respuesta es que viene de dos personas incluidas en grupos sociales diferentes: una ha sido pastor durante toda la vida y sigue viviendo en la zona (grupo A); tiene más de 70 años. La otra ha trabajado en el sector

Tabla 5. Influencia del proceso de revegetación en los aprovechamientos del territorio

\begin{tabular}{lccc}
\hline \multirow{2}{*}{ Aprovechamiento } & \multicolumn{2}{c}{ Sí influye } & \\
\cline { 2 - 3 } \multicolumn{1}{c}{ En la agricultura } & Favorable & Desfavorable & No influye \\
En la ganadería & 2 & & 23 \\
En la disponibilidad de pastos & 2 & 21 & 2 \\
En el turismo & 4 & 18 & 3 \\
En el ocio (visitas de 1 día) & 16 & 2 & 7 \\
En el bosque (madera) & 17 & 3 & 5 \\
En la caza & 4 & & 21 \\
En los subproductos del bosque & 18 & 4 & 3 \\
En el paisaje & 13 & 9 & 3 \\
En la recuperación de los pueblos & 18 & 7 & 20 \\
\hline
\end{tabular}

Fuente: elaboración propia a partir de entrevistas semiestructuradas. 
industrial con cargo de responsabilidad en una empresa y pertenece al grupo C; se incluye en el estrato de 60-70 años.

Resulta lógico que la mayoría de la población opine que la revegetación apenas tiene consecuencias sobre la agricultura, ya que ésta es prácticamente inexistente. Lasanta et al. (2011) señalan que a principios del siglo xx el espacio cultivado alcanzaba 15.491 ha (el 34,4\% de la superficie de Cameros Viejo), mientras que en 2006 sólo se cultivaban 54,3 ha (el 0,12\%), dedicadas a pequeños huertos en las proximidades de algunos pueblos.

La respuesta fue casi unánime sobre la relación entre revegetación y ganadería: 21 personas (el 84\%) respondieron que la influencia es negativa, ya que los matorrales impiden el acceso del ganado a los pastos del estrato inferior. Dos entrevistados (8\%) opinaron que es favorable al diversificar la oferta de pasto, sobre todo para el cabrío, que prefiere combinar el consumo de brotes de matorral con las herbáceas. Otros 2 entrevistados dijeron que la revegetación no tiene consecuencias sobre la ganadería porque la pérdida de pastos por el cubrimiento de los matorrales es compensada por los desbroces de la Administración.

Respecto a la disponibilidad de pastos para el ganado, 18 personas (el 72\%) consideran que ahora hay menos pastos porque «donde hay matorrales o pinos no crece la hierba», o porque los matorrales impiden el acceso del ganado al pasto. Uno de los interlocutores matiza que «en realidad hay más pasto que cuando se cultivaba, pero que está debajo de los matorrales y que, por ello, el ganado no lo puede consumir». Cuatro personas (16\%) opinan que ahora hay más pasto, ya que, según una de ellas, el abandono de tierras implica aumentar la superficie de pastos, y 3 interlocutores señalan que ahora hay menos ganado que antes, por lo que el pasto es más abundante. Los desbroces de la Administración llevan a que 2 personas declaren que la revegetación no afecta en nada a la disponibilidad y calidad de los pastos. Otro entrevistado dice que depende de lo que llueva: «en años de copiosas lluvias sobran pastos, mientras que si son escasas faltan».

Es muy evidente que una cubierta densa de matorrales disminuye la producción herbácea en unos casos (bajo Cistus laurifolius, por ejemplo), mientras que en otros impide el posible aprovechamiento por parte del ganado, ya que el estrato de matorral cubre unos pastos ricos, como ocurre bajo Genista scorpius (Lasanta, 2005). Por ello, el desbroce de aliagares implica pasar de una producción de $4.566 \mathrm{Mj} / \mathrm{ha} /$ año a $10.167 \mathrm{Mj} / \mathrm{ha} / \mathrm{año}$, lo que supone multiplicar la producción por 2,22, mientras que el desbroce de jarales implica pasar de $2.435,2 \mathrm{Mj} / \mathrm{ha} /$ año a $7.628,6 \mathrm{Mj} / \mathrm{ha} / \mathrm{año}$, es decir, un incremento por 3,13. Además, hay que tener en cuenta que la producción de aliagares y jarales sin desbrozar es potencial pero no real, ya que el ganado no tiene acceso al estrato herbáceo. Por otro lado, los matorrales sólo son aprovechados por el cabrío y el equino, si se encuentra en un ecotono con pastos herbáceos o en los márgenes de caminos y senderos (Lasanta et al., 2013).

Las opiniones estuvieron más divididas respecto al turismo: 16 personas (el 64\%) opinan que favorece la llegada de turistas, porque el paisaje es más bonito 
ahora que antes. La mayoría de este grupo manifestó que es en primavera, por la abundancia de flores, cuando el paisaje es más atractivo. Siete entrevistados (28\%) opinaron que la revegetación no tiene ningún efecto, coincidiendo todos ellos en dos premisas: i) Cameros Viejo no es una zona turística y ii) el paisaje — ni el tradicional ni el actual — tiene suficiente gancho para atraer a los turistas. Sólo 2 entrevistados opinaron que la evolución del paisaje es negativa para la actividad turística, considerando que el paisaje tradicional estaba más cuidado y que las laderas abancaladas, de haberse conservado en buen estado, serían un buen cebo para el turismo. Los entrevistados que consideraron que la revegetación era buena para el turismo, también opinaron que lo era para el ocio o visitas de un día.

Sólo 4 personas (16\%) opinaron que el avance de matorrales y árboles es beneficioso para el aprovechamiento maderero del bosque, aunque 2 de ellos concretaron que no en el presente pero sí en el futuro. Veintiún entrevistados $(84 \%)$ dieron la respuesta contraria, señalando mayoritariamente que el bosque no ha sido un recurso económico de la zona, ni lo será en el futuro, ya que tiene difícil competir con otras áreas más favorables para la producción de madera.

Respecto a la actividad cinegética, 18 personas (el 72\%) consideraron que el incremento de la cubierta vegetal es positivo para la caza, aunque varios de ellos especificaron que sobre todo para la caza mayor, mientras que para la menor podía ser indiferente o perjudicial. Según varios de los entrevistados algunas especies se verían beneficiadas con un paisaje «más vestido» porque tienen más áreas de refugio y más posibilidades de alimentación. Cuatro personas (16\%) dieron la respuesta contraria, señalando que el cese del cultivo implicó el descenso de las posibilidades de alimentación para muchas especies cinegéticas (jabalíes, ciervos), obligándolas a emigrar hacia los márgenes de zonas agrícolas. Tres entrevistados (12\%) opinaron que la caza no se ve afectada por los cambios de vegetación.

Las estadísticas oficiales del Gobierno de La Rioja señalan que tanto la fauna silvestre como el número de animales de caza mayor capturados siguen una tendencia ascendente durante las últimas décadas. Los animales capturados han pasado de 603 en la campaña 1988-89 a 5.735 en la campaña 2010-11. Los jabalíes eran los que más se cazaban inicialmente; en los últimos años, jabalíes y ciervos se capturan en proporciones parecidas, mientras que los corzos se cazan menos, si bien presentan también una evolución positiva.

Las opiniones están muy divididas respecto al papel de la revegetación en los subproductos del bosque, lo que parece lógico si tenemos en cuenta la amplia gama de productos obtenidos: el 52\% (13 personas) opinan que es favorable, señalando unos que la mayor presencia de árboles mejora la producción de setas; otros opinan que la abundancia de matorrales favorece la apicultura; otros que se podrían aprovechar leñas. El 36\% (9 personas) tienen la opinión contraria, señalando que los matorrales (sobre todo los jarales) impiden — para unos- el que haya subproductos (sobre todo setas), y - para otros- el acceso a ellos. Por último, el 12\% (3 personas) opinan que la revegetación es indife- 
rente para el aprovechamiento de los subproductos del bosque, señalando que no existe comercialización ni interés en desarrollarla.

Todos los entrevistados opinan que la revegetación tiene efectos sobre el paisaje. Las respuestas están llenas de matizaciones; así, por ejemplo, varios de los entrevistados dijeron que el paisaje ahora es más bonito, pero a continuación señalan que lo cubierto por bosques, pero no las laderas de matorrales. Por otro lado, son varios los que especifican que ahora es más bonito que el de décadas precedentes, cuando ya no había cultivos y sí grandes extensiones de suelo desnudo. Los que así opinan manifiestan también que el paisaje sería más atractivo si se hubieran conservado las estructuras agrícolas tradicionales (bancales, setos, corrales, sendas, caminos). Otros dicen que ellos no recuerdan el paisaje agrícola y sí otro de amplias superficies de suelo desnudo, muretes caídos y procesos de erosión muy extendidos, por lo que consideran el actual paisaje más atractivo, pero tienen dudas de cuál sería su respuesta si la comparación se produjera con el paisaje durante la fase de plena actividad agrícola. Las personas incluidas en el grupo A son las que opinan de forma más rotunda que el paisaje tradicional era más bonito, recalcando varios que «lo era porque estaba más limpio». El grupo $\mathrm{B}$ contesta mayoritariamente que ahora el paisaje es más bonito, sin entrar en matizaciones. El grupo $\mathrm{C}$ es el que tiene más dudas en la respuesta: si es un emigrante de cierta edad aclara que si el paisaje agrícola tradicional se hubiera conservado sería más atractivo que el actual, mientras que entre los más jóvenes y foráneos las matizaciones se establecen entre árboles y matorrales; los bosques son preferidos a las áreas de matorral.

Hay una estrecha relación entre los que consideran que ahora el paisaje es más bonito y los que opinan que la revegetación es favorable para el turismo y para el ocio. Sin embargo, algunos de los que opinaron que el incremento de la cubierta vegetal es negativo para el paisaje consideran que también afecta negativamente a la llegada de turistas; otros, sin embargo, se decantan por la indiferencia o por estimar que no tiene demasiados efectos.

Varios estudios señalan que la naturalidad puede resultar positiva para que la población considere un paisaje más bonito y más atractivo para visitar (Nassauer 2011; Arriaza et al., 2004; Rogge et al., 2007; Nijnik y Mather, 2008). Hunziker et al. (2008) observaron una actitud positiva entre los suizos hacia escenarios de sucesión forestal espontánea. Bauer et al. (2009) señalan, a partir de una encuesta en Suiza, que las preferencias sobre la renaturalización del paisaje cambian entre la población rural y urbana. Las personas que crecieron en el campo tienen una perspectiva más negativa. A idénticos resultados llegaron Höchtl et al. (2005) en estudios realizados en los Alpes italianos y Ruskule et al. (2013) en Letonia.

La mayoría de los entrevistados (20 personas, el $80 \%$ ) se decanta por opinar que la revegetación no tiene efectos en la recuperación de los pueblos de la zona, si bien 4 personas opinan que es un factor positivo, ya que la gente valora más un paisaje más verde. Un interlocutor señala que es negativo porque «la existencia de tanta maleza (matorral) da la sensación de descuido». 


\subsection{Impactos en el medio natural}

La tabla 6 trata de responder a los impactos de la revegetación en el medio natural y en las actividades socioeconómicas. Las opiniones son bastante unánimes (el 92\%) en que el mayor cubrimiento de vegetación implica una mejor conservación del suelo. Son muchos los que opinan que las aguas de escorrentía salían más cargadas en la fase agrícola que en la actual. No obstante, una persona de 83 años, que emigró a Logroño hace 40 años, contesta que «antes había menos erosión porque el agricultor se encargaba de mantener sus campos; ahora, sin embargo, los muretes de los bancales se desploman y hay más cicatrices en muchas parcelas». Otra persona señala que los cambios en la cubierta vegetal son menos importantes que los registrados en las precipitaciones. Antes — dice- «había más nublados y, por ello, había más erosión». García-Ruiz y Lana-Renault (2011) concluyen, a partir de una revisión bibliográfica para toda Europa, sobre qué implicaciones tiene el abandono de tierras y la revegetación posterior en la erosión del suelo, que el incremento de la vegetación lleva a que las tasas de erosión del suelo disminuyan. Ello es una consecuencia lógica del efecto protector de la vegetación contra el salpicado de la lluvia y el desprendimiento de partículas. Además, las características del suelo tienden a mejorar, lo que favorece la infiltración del agua y la estabilidad de los agregados.

La respuesta es unánime respecto al riesgo de génesis y propagación de incendios: todos opinan que ahora hay más probabilidad de que haya incendios catastróficos porque hay mucha maleza en el monte. Antes, por el contrario, el monte estaba más limpio y se cogía mucha leña (árboles muertos, ramas caídas...). Además, los matorrales — sobre todo las jaras - se utilizaban en hornos y hogares, e incluso se intercambiaban por paja con gentes del llano. Por último, son bastantes los que opinan que los pequeños fuegos realizados en el pasado por los ganaderos controlaban la expansión de los matorrales y, de esta forma, ayudaban a evitar grandes incendios.

El incremento de los incendios y de la superficie quemada se ha explicado en ocasiones como una consecuencia del calentamiento global, especialmente en las regiones boreales, donde la estación de incendios tiende a durar más tiempo y ocupar superficies más amplias (Kasischke y Turetsky, 2006). Sin embargo, la mayor parte de la literatura pone el acento en los cambios de uso

Tabla 6. Impactos del proceso de revegetación en el medio natural

\begin{tabular}{|c|c|c|c|c|}
\hline & Hay & cto & & \\
\hline & Bueno & Malo & No hay & No sabe/indiferente \\
\hline En la erosión del suelo & 23 & 1 & 1 & \\
\hline En los incendios & & 25 & & \\
\hline En la cantidad de agua & 9 & 15 & & 1 \\
\hline En la calidad del agua & 19 & 3 & 2 & 1 \\
\hline En la fauna & 21 & 2 & & 2 \\
\hline
\end{tabular}

Fuente: elaboración propia a partir de entrevistas semiestructuradas. 
del suelo, especialmente en el abandono de tierras y el desaprovechamiento de recursos forestales y ganaderos, lo que lleva a un proceso de revegetación y al aumento del material combustible (Scarascia-Mugnozza et al., 2000; Chauchard et al., 2007; Serra et al., 2008; Pausas y Keeley, 2009). En España, por ejemplo, en la década que va de 1968 a 1977 se quemaron $892.066,5$ ha, mientras que la superficie ascendió a 1.437 .896 ha entre los años 2003 y 2012, a pesar de la mejora de los medios de prevención y extinción de incendios. La principal causa del incremento de los incendios es la existencia de mayor combustible, como consecuencia del abandono del medio rural (Pausas, 2010).

El 60\% de los entrevistados opina que ahora hay menos agua en las cuencas que en el pasado, señalando que "los ríos llevan ahora menos agua", «hay fuentes que han desaparecido", y que la causa de ello es que "la vegetación retiene y consume agua». El 36\% coincide en señalar que ahora hay más agua que en el pasado, explicándolo por la capacidad de la vegetación para regular y distribuir mejor el agua de lluvia; otros aluden a que ahora los acuíferos se recargan más y mejor y que «la vegetación genera más agua, porque el bosque atrae a las precipitaciones». Una persona señala que «si en la actualidad hay menos agua es porque llueve menos, no dependiendo para nada de la cubierta vegetal». Son mayoría (19 entrevistados; el 76\%) los que piensan que el agua que llevan los ríos o manan las fuentes es de mayor calidad que en el pasado, debido a que la vegetación la filtra, mientras que antes salía más directa, el tiempo de respuesta de la escorrentía era más corto. Tres personas (el 12\%) señalan que la calidad empeora, relacionándolo con la no limpieza de las fuentes, «cosa que antes se realizaba periódicamente».

Resulta curioso comprobar que el grupo A responde que ahora hay menos agua pero de mayor calidad. El grupo B tiene la opinión contraria. El grupo C muestra mayor diversidad de opiniones, si bien los emigrantes de mayor edad coinciden con el grupo A, mientras que los que proceden de fuera coinciden con la percepción del grupo B.

Los estudios experimentales muestran que la expansión de la cubierta vegetal produce caudales más bajos, por aumento de la interceptación de la lluvia, el incremento del consumo de agua por los bosques y matorrales, sobre todo en verano, y la mayor infiltración del agua en el suelo (Andréassian, 2004; Serrano-Muela et al., 2008). Gallart y Llorens (2004) estudiaron la evolución de los caudales en los afluentes del Ebro, comprobando una tendencia descendente, lo que atribuyen a la expansión y densificación de la cubierta vegetal. Otros estudios llegan a las mismas conclusiones (Beguería et al., 2003; MoránTejada et al., 2010).

Una elevada proporción de los actores piensa que la revegetación ha contribuido a que exista más fauna, especificando que sobre todo han aumentado los corzos, jabalíes y depredadores. Para 21 personas $(84 \%)$ que haya más fauna es positivo, mientras que 2 (ambos del grupo A) lo consideran negativo: una mujer porque hay más culebras y un señor porque "corzos y jabalíes se meten en los huertos, te estropean las plantas y te quitan las ganas de sembrar». Dos personas consideran que el incremento de la cubierta vegetal es indiferente para 
la fauna. Las estadísticas oficiales reflejan el incremento de la fauna silvestre en La Rioja. Otras regiones españolas han seguido la misma tendencia, especialmente en las dos últimas décadas, aumentando tanto la superficie ocupada por la fauna como el número de ejemplares, debido a su recuperación natural y a traslados efectuados entre distintas áreas de montaña (Bueno et al., 2011; Prada y Herrero, 2013).

\section{Conclusiones e implicaciones}

Los resultados cualitativos obtenidos en este trabajo ponen de relieve que los stakeholders de Cameros Viejo son conscientes del proceso de revegetación que tiene lugar tras el abandono de tierras y la emigración de la población. Además, su percepción coincide con los resultados cuantitativos aportados por las estadísticas oficiales y la literatura científica en cuanto a las principales causas del abandono de tierras (repoblación por parte de la Administración, falta de ganado y falta de personas en el monte), en algunas de sus consecuencias socioeconómicas (agricultura, ganadería, paisaje, caza), y en los impactos en el medio natural (erosión del suelo, riesgo de incendios, fauna silvestre y calidad del agua). Difieren en otras causas, consecuencias e impactos, lo que es habitual en este tipo de estudios. Fernández-Giménez y Fillat, (2012) señalan que es frecuente que algunas percepciones y conocimientos sean ampliamente compartidas por un grupo local, si bien en otras se producen diferencias en función de la edad, la experiencia, el género, el papel social de cada individuo en la comunidad...

Por otro lado, hay que destacar que la población residente (grupo A) tiene mejor percepción que la población foránea (grupos $\mathrm{B}$ y C) sobre los cambios recientes en el medio natural, lo que se deduce al comparar las respuestas de los entrevistados con los trabajos científicos realizados en el área de estudio o en áreas próximas de la montaña mediterránea. Este hecho, puede tener sus implicaciones, ya que el sector de población que mejor percibe los cambios es el de mayor edad y el que está perdiendo capacidad de intervenir en el territorio, mientras que los sectores más activos tienen una percepción distorsionada de algunas consecuencias socioeconómicas e impactos en el medio natural.

Las respuestas de los entrevistados aportan una visión que puede complementar los resultados de los científicos. Sugieren, además, nuevas investigaciones sobre los cambios de uso del suelo y sus implicaciones, así como sobre las causas que influyen en la expansión de matorrales y bosques. Animamos tanto a los científicos como a los gestores del territorio a colaborar más estrechamente con los actores del territorio. Asimismo, sugerimos que los residentes participen activamente en la toma de decisiones de mantenimiento de los recursos y adaptación al cambio global. La literatura especializada sostiene que la participación de los usuarios locales en las decisiones de supervisión y gestión es una forma de asegurar que las normas elaboradas se utilizan inmediatamente (Danielsen et al., 2010). En una época con cambios climáticos y socioeconómicos muy rápidos, la capacidad de detectar y responder 
adecuadamente a tales cambios determinará, probablemente, qué sistemas mantienen su capacidad de recuperación y cuáles cruzan los umbrales hacia un estado irreversible.

\section{Agradecimientos}

Este trabajo se ha realizado con el apoyo financiero de los proyectos: INDICA (CGL2011-27753-CO2-01 y 02) e HIDROCAES (CGL2011-27574CO2-02), financiados por el Ministerio de Economía y Competitividad (Gobierno de España).

\section{Referencias bibliográficas}

ANDréassian, V. (2004). «Waters and forest: from historical controversy to scientific debate». Journal of Hydrology, (291), 1-27.

Antrop, M. (2005). «Why landscape of the past are important for the future». Landscape and Urban Planning, 70 (1-2), 21-34.

- (2006). «Sustainable landscapes: Contradiction, fiction or utopia?». Landscape and Urban Planning, 75 (3-4), 187-197.

Arnáez, J.; Lasanta, T.; Errea, M. P. y Ortigosa, L. (2011). «Land abandonment, landscape evolution, and soil erosion in a Spanish Mediterranean mountain region. The case of Cameros Viejo». Land Degradation and Development, (22), 537-550.

Arnáez, J.; Ortigosa, L.; Oserín, M. y Lasanta, T. (2009). «Evolución de la cubierta vegetal en Cameros entre 1956 y 2001». En: Lasanta, T. y Arnákz, J. (eds.). Gestión, usos del suelo y paisaje en Cameros. Sistema Ibérico, La Rioja. Logroño: Universidad de La Rioja e Instituto de Estudios Riojanos: 127-144.

Arriaza, M.; Cañas-Ortega, J. F.; Cañas-Madueño, J. A. y Ruiz Avilés, P. (2004). "Assessing the visual quality of rural landscapes». Landscape and Urban Planning, 69 (1), 115-125.

Bauer, N.; Wallner, A. y Hunzinker, M. (2009). «The change of European landscapes: Human-nature relationships, public attitudes towards rewilding, and the implications for landscape management in Switzerland». Journal of Environmental Management, 90 (9), 2910-2920.

Beguería, S.; López-Moreno, J. I.; Lorente, A.; Seeger, M. y García-Ruiz, J. M. (2003). "Assessing the effect of climate oscillations and land-use changes on streamflow in the Central Spanish Pyrenees». Ambio, 32 (4), 283-286.

Bell, S.; Montarzino, A.; Aspinall, P.; Peneze, Z. y Nikodemus, O. (2009). «Rural society, social inclusion and landscape change in Central and Eastern Europe: A case study of Latvia». Sociologia Ruralis, 49 (3), 295-326.

Benjamin, K.; Bouchard, A. y Domon, G. (2007). «Abandoned farmland as components of rural landscapes: An analysis of perceptions and representations». Landscape and Urban Planning, 83 (4), 228-244.

Bowen, M. E.; McAlpine, C. A.; House, A. P. N. y Smith, G. C. (2007). «Regrowth forest on abandoned agricultural land: A review of their habitat values for recovering forest fauna». Biological Conservation, 140 (3-4), 273-296.

Bueno, C. G.; Barrio, I. C.; García-González, R.; Alados, C. L. y Gómez-GarCíA, D. (2011). «Assessment of wild boar rooting on ecological and pastoral values of alpine pyrenean grassland». Pirineos, (166), 51-67. 
Chauchard, S.; Carcaillet, C. y Guibal, F. (2007). «Patterns of land-use abandonment control tree-recruitment and forest dynamics in Mediterranean mountains». Ecosystems, (10), 936-948.

Danielsen, F.; Burgess, N. D.; Jensen, P. M. y Pirhofer-Walzl, K. (2010). «Environmental monitoring: the scale and speed of implementation varies according to the degree of people's involvement». Journal of Applied Ecology, (47), 1166-1168.

Domon, G. (2011). "Landscape as resource: Consequences, challenges and opportunities for rural development». Landscape and Urban Planning, 100 (4), 338-340.

Fernández-Giménez, M. E. y Fillat, F. (2012). «Pyrenean pastoralists' observations of environmental change: an exploratory study in los Valles Occidentales of Aragon». Pirineos, (167), 143-163.

Gallart, F. y Llorens, P. (2004). «Observations on land cover changes and water resources in the headwaters of the Ebro catchment, Iberian Peninsula». Physics and Chemistry of the Earth, (29), 769-773.

García-Ruiz, J. M. y Lana-Renault, N. (2011). «Hydrological and erosive consequences of farmland abandonment in Europe, with special reference to the Mediterranean region - A review». Agriculture, Ecosystems and Environment, (140), 317-338.

Gaviría, M. y Baigorri, A. (1984). El campo riojano. Logroño: Cámara Agraria Provincial, 2 Tomos.

Gellrich, M.; Baur, P.; Koch, B. y Zimmermann, N. E. (2007). «Agricultural land abandonment and natural forest re-growth in the Swiss mountains: A spatially explicit economic analysis». Agriculture, Ecosystems and Environment, 118 (1-4), 93-108.

Henle, K.; Alard, D.; Clitherow, J.; Cobb, P.; Firmank, L.; Kull, T. et al. (2008). "Identifying and managing the conflicts between agriculture and biodiversity conservation in Europe - A review». Agriculture, Ecosystems and Environment, 124 (1-2), 60-71.

Höchtl, F.; Lehringer, S. y Konold, W. (2005). "Wilderness”: What it means when it becomes a reality - A case study from the southwestern Alps». Landscape and Urban Planning, 70 (1-2), 85-95.

Hunziker, M.; Felber, P.; Gehring, K.; Buchecker, M.; Bauer, N. y Kienast (2008). «Evaluation of landscape change by different social groups: Results of two empirical studies in Switzerland». Mountain Research and Development, 28 (2), 140-147.

Kasischie, E. S. y Turetsky, M. R. (2006). «Recent changes in the fire regime across the NortAmerica boreal region - Spatial and temporal patterns of burning across Canada and Alaska». Geophysical Research Letters, (33), 1-5.

Kaur, E.; Palang, H. y Sooväli, H. (2004). «Landscapes in change - Opposing attitudes in Saaremaa, Estonia». Landscape and Urban Planning, 57 (1-4), 109-120.

Keenleyside, C. y Tucker, G. M. (2010): Farmland abandonment in the EU: an assessment of trends and prospects. Report prepared for WWF. Londres: Institute for European Environmental Policy: 93 pp.

Lasanta, T. (2005). "Gestion des champs abandonnés pour le dévelopment de l'élevage extensif dans les Pyrénées centrales espagnoles ». Sud-Ouest Européen, (19), 109-119.

Lasanta, T. (2009). «La ganadería en Cameros: entre la adaptación a los recursos y la dependencia del exterior. En: Lasanta, T. y Arnáez, J. (eds.) Gestión, usos del suelo y paisaje en Cameros. Sistema Ibérico, La Rioja. Logroño: Universidad de La Rioja e Instituto de Estudios Riojanos, 191-222. 
Lasanta, T. y Errea, M. P. (2001). Despoblación y marginación en la Sierra Riojana. Logroño: Instituto de Estudios Riojanos, $181 \mathrm{pp}$.

Lasanta, T.; Errea, M. P.; Vicente-Serrano, S. M. y Arnáez, J. (2011). «La diversidad de la cubierta vegetal en campos abandonados del Leza y Jubera (Sistema Ibérico, La Rioja) a partir del SIOSE». Zubia. Monográfico (23), 55-78.

Lasanta, T.; Errea, M. P.; Bouzzebboudja, M. R. y Medrano, L. M. (2013). Pastoreo y desbroce de matorrales en Cameros Viejo. Logroño: Instituto de Estudios Riojanos. Colección Ciencias de la Tierra, 30, 186 pp.

Lasanta, T. y Vicente-Serrano, S. M. (2003). «Evolución del paisaje en la montaña submediterránea durante la segunda mitad del siglo xx: un caso de estudio en la cuenca del Jubera». Zubía. Monográfico (13), 193-210.

Lasanta, T. y Vicente-Serrano, S. M. (2007). "Cambios en la cubierta vegetal en el Pirineo Aragonés en los últimos 50 años». Pirineos, (162), 125-154.

Lasanta, T.; Vicente-Serrano, S.M. y Cuadrat, J. M. (2000). "Marginación productiva y recuperación de la cubierta vegetal en el Pirineo: un caso de estudio en el valle de Borau». Boletín de la Asociación de Geógrafos Españoles, (29), 5-28.

Lasanta, T.; Vicente-Serrano, S. y Cuadrat, J. M. (2005). "Mountain Mediterranean landscape evolution caused by the abandonment of traditional primary activities: a study of the Spanish Central Pyrenees». Applied Geography, (25), 47-65.

MacDonald, D.; Crabtree, J. R.; Wiesinger, G.; Dax, T.; Stamou, N.; Fleury, P.; Gutiérrez-Lazpita, J. y Gibon, A. (2000). "Agricultural abandonment in mountain areas of Europe: environmental consequences and policy response». Journal Environmental Management, (59), 47-69.

Molinillo, M.; Lasanta, T. y García-Ruiz, J. M. (1997). «Managing degraded landscapes after farmland abandonment in the Central Spanish Pyrenees». Environmental Management, (21), 587-598.

Morán-Tejada, E.; Ceballos-Barbancho, A. y Llorente-Pinto, J. M. (2010). «Hydrological response of Mediterranean headwaters to climate oscillations and land-cover changes: the mountains of Duero River basin (Central Spain)». Global and Planetary Change, (72), 39-49.

Nassauer, J. I. (2011). "Care and stewardship: From home to planet». Landscape and Urban Planning, 100 (4), 321-323.

Navarro, L. M. y Pereira, H. M. (2012). «Rewilding abandoned landscapes in Europe». Ecosystems, 15: 900-912.

Nijnik, M. y MAther, A. (2008). «Analyzing public preferences concerning woodland development in rural landscapes in Scotland». Landscape and Urban Planning, 86 (3-4), 267-275.

Ortigosa, L. (1990). «Las repoblaciones forestales como estrategia pública de intervención en regiones degradadas de montaña». En: García-Ruiz, J. M. (ed.). Geoecología de las áreas de montaña. Logroño: Geoforma Ediciones: 297-311.

Ortigosa, L., García-Ruiz, J. M. y GiL, E. (1990). «Land reclamation by reforestation in the Central Pyrenees». Mountain Research and Development, 10 (3), 281-288.

Pausas, J. G. (2010). «Fuego y evolución en el Mediterráneo». Investigación y Ciencia, (407), 56-63.

Pausas, J. G. y Keeley, J. E. (2009). «A burning story: the role of fire in the history of life». Bioscience, 59 (7), 593-601.

Prada, C. y Herrero, J. (2013). «Viabilidad del hábitat y del reforzamiento poblacional de Capra Pirenaica en la Reserva nacional de Caza os Ancares». Pirineos, (168), 59-76. 
Rogge, E.; Nevens, F. y Gulinck, H. (2007). "Perception of rural landscapes in Flanders: Looking beyong aesthetics». Landscape and Urban Planning, 82(4), 159174.

Ruskule, A.; Nikodemus, O.; Kasparinskis, R.; Bell, S. y Urzane, I. (2013). «The perception of abandoned farmland by local people and experts: landscape value and perspectives on future land use». Landscape and Urban Planning, (115), 49-61.

Sayadi, S.; González-Roa, M. C. y Calatrava-Requena, J. (2009). "Public preferences for landscape features: The case of agricultural landscape in mountainous Mediterranean areas». Land Use Policy, 26 (2), 234-344.

Scarascia-Mugnozza, G.; Oswald, H.; Piussi, P. y Radoglou, K. (2000). «Forest of the Mediterranean region: gaps in Knowledge and research needs». Forest Ecology Management, (132), 97-109.

Serra, P.; Pons, X. y Saurí, D. (2008). «Land-cover and land-use change in a Mediterranean landscape: a spatial analysis of driving forces integrating biophysical and human factors». Applied Geography, 28 (3), 189-209.

Soliva, R. (2007). "Landscape stories: Using ideal type narratives as heuristic device in rural studies». Journal of Rural Studies, (23), 62-74.

Stoate, C.; Báldi, A.; Beja, P.; Boatman, N. D.; Herzon, I. van Doorn, A. et al. (2009). "Ecological impacts of early $21^{\text {st }}$ agricultural change in Europe - A review». Journal of Environmental Management, 91 (1), 22-46.

Uematsu, Y.; Koga, T.; Mitsuhashi, H. y Ushimaru, A. (2010). «Abandonment and intensified use of agricultural land decrease habitats of rare herbs in semi-natural grasslands». Agriculture, Ecosystems and Environment, 135 (1-2), 304-309.

Vicente-Serrano, S.; Lasanta, T. y Romo, A. (2005). "Analysis of spatial and temporal evolution of vegetation cover in the Spanish Central Pyrenees: Role of Human Management. Environmental Management, (34), 802-818.

Waisamen, P. J. y Bliss, N. B. (2002). «Changes in population and agricultural land in conterminous United States counties, 1790-1997». Global Biogeochemical Cycles, (16), 1-19. 